\title{
Water monitors; Implications in forensic death investigations.
}

\author{
Gunethilake KMTB ${ }^{1}$, Vidanapathirana $\mathrm{M}^{2}$
}

\begin{abstract}
Introduction: Outdoor postmortem animal scavenging is frequent and the predators responsible vary from ants, foxes, bluebottles to monitor lizards. Postmortem scavenging by water monitors is not uncommon. The water monitor (Varanus salvator) is a large lizard native to South East Asia. The sub species V.s salvator, is limited to Sri Lanka and are efficient carnivores and scavengers. Therefore, there is significant impact on forensic death investigations

Case report: Case 1: A woman went missing. A vigorous search lead to the recovery of the body in a river, surrounded by water monitors. Injuries on the limbs simulated cut injuries. The skull had a depressed slashed cut exposing brain matter. Case 2: The dead body was located by searching along a path formed by water monitors. The neck injuries simulated cut throat. These two cases are discussed to highlight the medico-legal implications of water monitors in forensic death investigations.

Conclusion: The presence of water monitors can be helpful in locating a missing body. However, it is necessary to interpret postmortem features cautiously in such cases as injuries caused by water monitors may resultin artifacts, make injury interpretation difficult, make identification difficult, pose challenges to ascertaining the cause of death, and move objects from the scene.
\end{abstract}

Key words: water monitor, outdoor scavenging, advantages, disadvantages

\section{Full paper}

\section{Introduction}

Outdoor postmortem animal scavenging is encountered frequently ${ }^{[1]}$ and the predators varying from ants to foxes and from bluebottles to monitor lizards. ${ }^{[2]}$ Of them, postmortem scavenging by water monitors is not uncommon. ${ }^{[3]}$ Water monitor (Varanus salvator) is a large lizard native to South East Asia. They are $1.5-2 \mathrm{~m}$ in size and the largest water monitor was recorded from Sri Lanka. ${ }^{[4]}$ They live closer to water. The sub species V.ssalvator, commonly called as 'Kabaragoya' is limited to Sri Lanka and are found in every environment. ${ }^{[5]}$ Adult water monitors are found in drier forests while young water monitors frequently live vicinity of the rivers. ${ }^{[5]}$

${ }^{1}$ Consultant Judicial Medical Officer, Provincial General Hospital, Ratnapura, ${ }^{2}$ Professor, Department of Forensic Medicine, Faculty of Medical Sciences, University of Sri Jayewardenepura, Sri Lanka.

*Corresponding author: Vidanapathirana M, email: mudithavidana@sjp.ac.lk, Tel: 0094772988227

http://orcid.org/0000-0003-0071-0996

DOI: http://doi.org/10.4038/mljsl.v4i2.7338
They are efficient carnivorous and scavengers. They eat dead bodies found in any environment irrespective of ground or in water ${ }^{[5]}$ and cause many effects on forensic death investigations. Further, postmortem scavenging of outdoor dead bodies by water monitors is not uncommon in day to day forensic practice and are one of the medico-legally important animals in Sri Lanka.

\section{Case report}

Case 1: A woman went missing and was suspected of being disposed in water. A vigorous search was done by hundreds of villagers and the Navy. The body was ultimately recovered from a river due to the abundance of water monitors attracted to the scene (Fig. 1). The body was in moderate state of putrefaction. There were multiple almost parallel tears and cuts in addition to the large losses of flesh with teeth marks. Injuries on the limbs (Fig. 2) simulated cut injuries. However, the skull bones had a depressed through and through slashed cut of the skull (Fig. 3).The cause of death was head injuries due to sharp force trauma. 


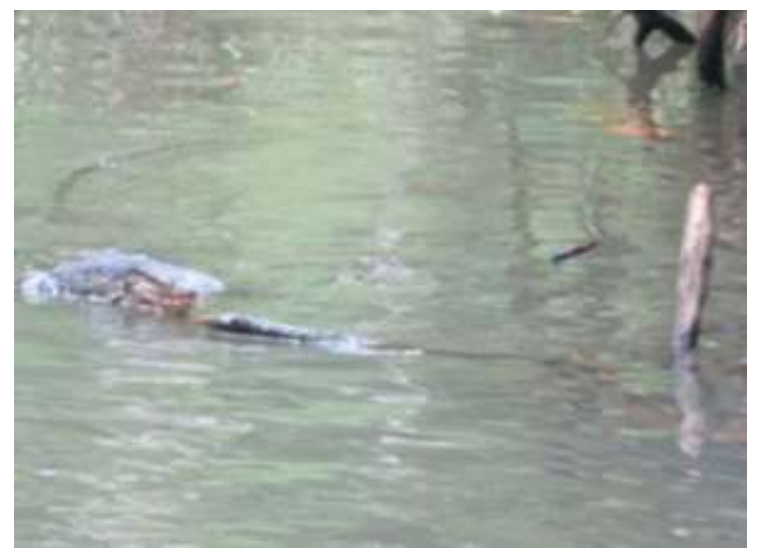

Figure 1. Abundance of water monitors indicated the site of the dead body.

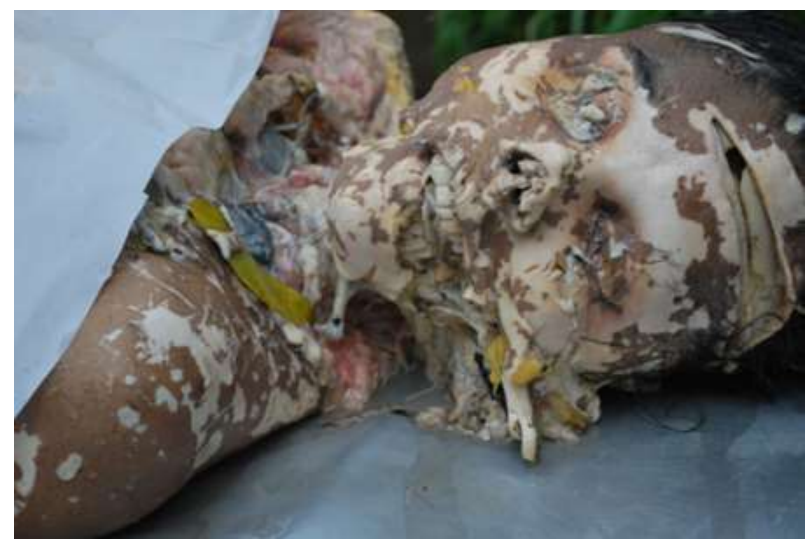

Figure 3. Through and through slashed cut of the skull.

Case 2: In another case, the site of the dead body was identified in a growing paddy field when searched along the path formed by the water monitors (Fig. 4 and 5). Body was in moderate state of putrefaction and had tears, cuts and teeth marks on the body. The neck injuries simulated cut throat (Fig. 6). However, there

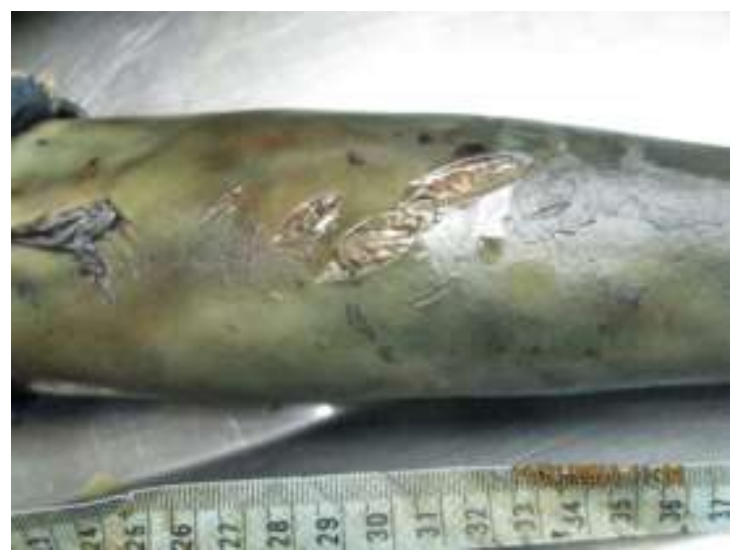

Figure 2. Limb injuries simulated cuts was damage and loss of internal organs and the cause of death was unascertainable.

These two cases are discussed to highlight the medicolegal implications of water monitors in forensic death investigations. 


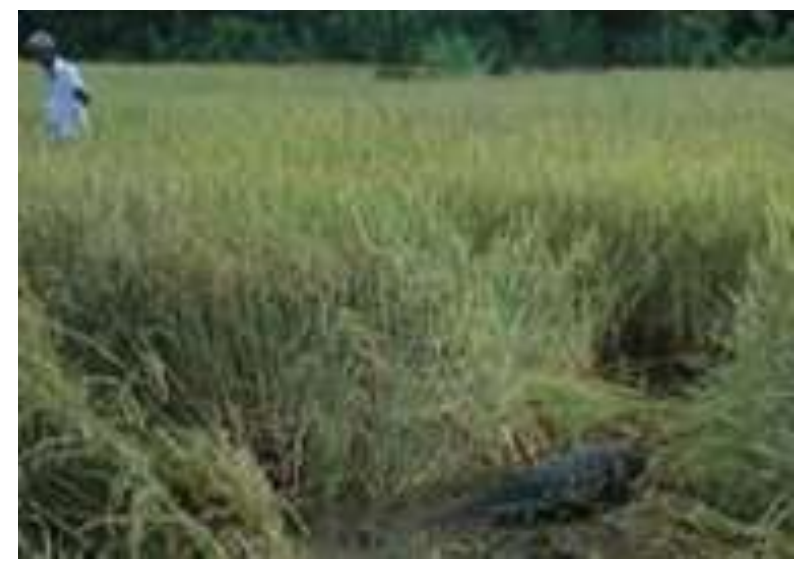

Figure 4. Followed the path of water monitors.

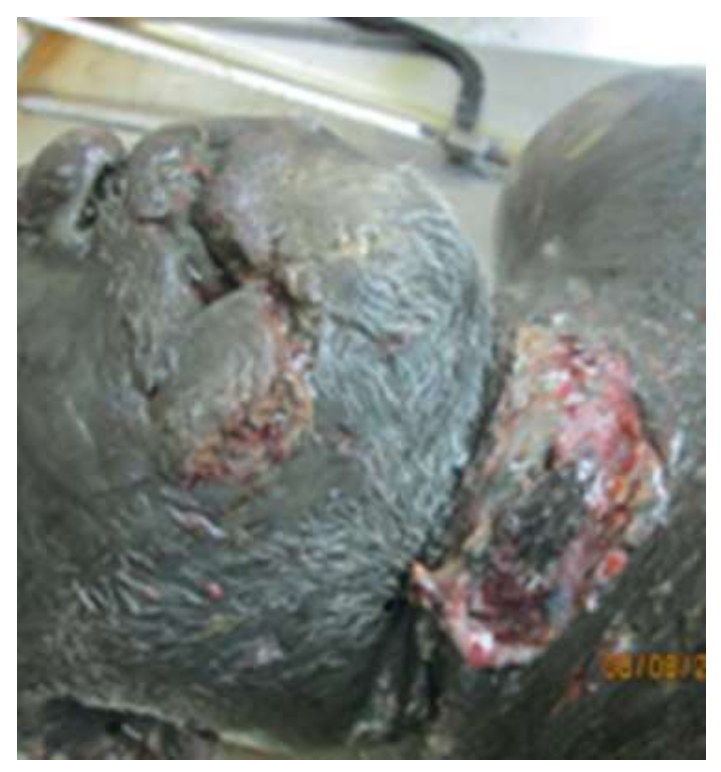

Figure 6. Neck injuries simulated cut throat

\section{Discussion}

Water monitors (Varnus salvator) prefer a fresh carcass to a rotten one and effects of water monitors on forensic death investigations is vast. It affects the scene, the body and the finding of the autopsy.

It is not always disadvantageous and in some circumstances, they are useful to identify the site of disposal of the dead body. When bodies are disposed in water, it is difficult to find. But water monitors are sensitive to smell and attract early. They follow a scent trail and scent a rotten carcass up to $100 \mathrm{~m}$ away. Irrespective of ground or water they are attracted to the scene. However, they only feed on carrion if found within their home range. ${ }^{[3]}$

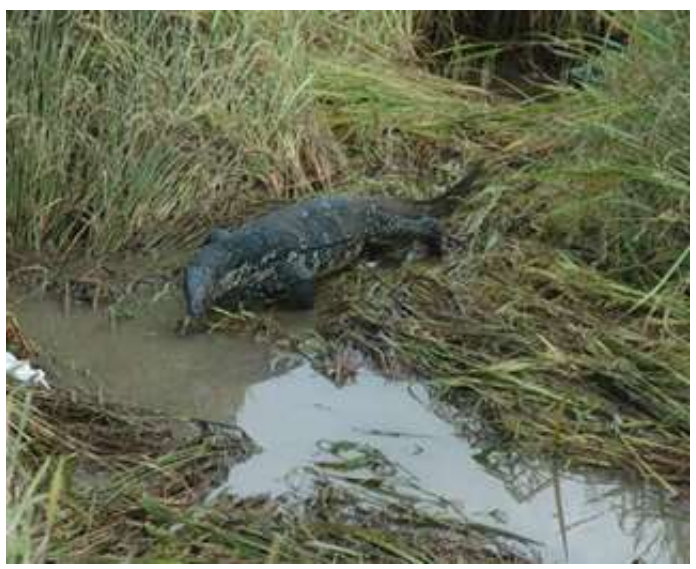

Figure 5. Ultimately found the site of the body

Water monitors swim using their long tail. ${ }^{[6]}$ In Case 1 , a women was killed and hide in water, a vigorous search was done but the body was ultimately recovered from a river due to the abundance of water monitors attracted to the scene (Figure 1).

Incase 2, the body was recovered from a growing paddy field when searched along the path formed by the water monitors (Fig. 4 and 5). Kabaragoyas can move fast and steadily even in muddy locations because the lateral bending during locomotion stabilizes the trunk. ${ }^{[7]}$ 
Water monitors affect the death scene findings too. They are relatively large animals and because of their movements at the scene and dragging the items or body away from original locus, the scene converts suspicious. Further, dealing with water monitors at the scene can be a terrifying experience to the forensic pathologists, even they are not much harmful.

The importance of water monitors in forensic death investigations in Sri Lanka was first reported by the authors. ${ }^{[8]}$ The postmortem injuries caused by water monitors can affect the interpretation of the autopsy findings. Those postmortem artifacts can simulate anti-mortem injuries ${ }^{[9]}$ and the differentiation of postmortem injuries from the ante-mortem injuries is important. In Case 1 and 2, the multiple, almost parallel tears with no vital reaction were compatible with animal predation and the severity of the cuts and the presence of water monitors around the scene suggested that those were teeth and claw injuries of Kabaragoyas. They have sharp claws which insert post mortem artifacts like incised injuries or stabs. Further, in Case 2, the areas with large tissue losses such as in neck were accompanied by teeth marks. Though the injuries of the limbs in Case 1simulatedcut injuries and injuries on the neck in Case 2simulated cut throat, the presence of associated teeth and claw marks helped to differentiate and identify them as postmortem animal bites. ${ }^{[2]}$

Further, the identification of antemortem injuries among postmortem animal injuries is also important, especially to ascertain the cause of death. There are no vital signs such as active haemorrhage in to the wound margin and reddening or oedema of the edges in postmortem injuries. ${ }^{[2]}$ In Case 1 , since it had a deep slashed cut in the head with underlying depressed, through and through skull cut indicated antemortem chop with a sharp, long, heavy, hard, rigid weapon such as manna or sward. However, since the body was in a state of moderate putrefaction and was recovered from water, this scalp cut did not show vital reactions and simulated postmortem animal bites. Therefore, when differentiating antemortem and postmortem injuries, the internal injuries should also be considered. Therefore, the cause of death in Case 1 was ascertained as head injury due to sharp force trauma.

As seen in Case 1 and 2, water monitors eat away flesh and tear off clothing making the identification difficult. They breach the skin barrier enhancing putrefaction by facilitating invasion of microorganisms and maggots. Most importantly they eat internal organs making decision of cause of death difficult and histopathology impossible. In some of the cases, authors have observed that all the chest organs have been eaten by water monitors entering through thoracic outlet. Similarly, in case 2, the cause of death was unascertainable especially due to postmortem animal predation.

The flesh of water monitor is not eaten, due to the belief in our culture that its flesh is highly poisonous. ${ }^{[5]}$ further, there is another belief that oil extracted from them is used for deliberate poisoning. That also makes the water monitor a significant animal in forensic death investigations.

\section{Conclusion}

The presence of water monitors can be helpful in locating a missing body. However, it is necessary to interpret postmortem features cautiously in such cases as injuries caused by water monitors may result in artifacts, make injury interpretation difficult, make identification difficult, pose challenges to ascertaining the cause of death, and move objects from the scene and convert it suspicious.

\section{References}

1. Weeraratna JB, Amararatne S, Ranasinghe RASK, Vidanapathirana M. Indoor postmortem animal scavenging - a case report. Sri Lanka Journal of Forensic Medicine, Science \& Law. 2014; 5 (1): 2-8.

2. Saukko P, Knight B. Post-mortem damage by the predators. B. Knight's Forensic Pathology, $3^{\text {rd }}$ Ed. CRC press, Boca Raton: USA; 2004. Pp 73-76.

3. Traeholt C. Notes on the water monitor Varanus salvator as scavenger. Malayan Nature Journal (Malaysia), 1994; 47 (3): 345-353.

4. Shine, R, Harlow PS, Keogh JS. "Commercial harvesting of giant lizards: The biology of water monitors Varanus salvator in southern Sumatra". Biological Conservation. 1996; 77 (2-3): 125134.

5. Family Varanidae (Monitor lizards). http://www.srilankanreptiles.com/Tetrapod

Reptiles /Varanidae.html. Retrieved on 10.02.2016.

6. Young BA, Dumais J, John N, Lyons B, Macduff A, Most M, Reiser NA, Reiser PJ. Functional Segregation within the Muscles of Aquatic Propulsion in the Asiatic Water Monitor (Varanus 
salvator). Frontiers in Physiology. 2016. 8;7:380. doi: 10.3389/fphys.2016.00380. eCollection 2016.

7. Ritter D. Axial muscle function during lizard locomotion. Journal of Experimental Biology. 1996;199 (Pt 11):2499-510.

8. Gunethilake KMTB, Vidanapathirana M. Water monitors: Importance in forensic death investigations. Conference paper: Annual academic sessions of the College of Forensic Pathologists of Sri Lanka, January 2016.

9. Rothschild MA, Schneider V. On the temporalonset of post-mortem animalscavenging. "Motivation" of the animal. Forensic Science International. 1997 Sep 19;89(1-2):57-64. 